Research Article

\title{
Research Activity among Academic Medical Staff during the COVID-19 Pandemic in Marrakesh
}

\author{
Latifa Adarmouch $\left(\mathbb{D}\right.$, Majda Sebbani $\left(D\right.$, and Mohamed Amine ${ }^{(D)}$ \\ Clinical Research Department, Mohammed VI University Hospital, Marrakesh, Morocco \\ Community Medicine and Public Health Department, Bioscience et Santé Research Lab, School of Medicine, \\ Cadi Ayyad University, Marrakesh, Morocco \\ Correspondence should be addressed to Latifa Adarmouch; 1.adarmouch@hotmail.com
}

Received 8 October 2020; Revised 17 November 2020; Accepted 24 November 2020; Published 7 December 2020

Academic Editor: Gwo-Jen Hwang

Copyright (C) 2020 Latifa Adarmouch et al. This is an open access article distributed under the Creative Commons Attribution License, which permits unrestricted use, distribution, and reproduction in any medium, provided the original work is properly cited.

\begin{abstract}
Introduction. The COVID-19 pandemic has profoundly impacted the medical academic institutions and their activities. Our aim was to describe the research activity (COVID-19-related or preexisting research) of the academic staff at the medical school in Marrakesh, Morocco. Methodology. An online survey among faculty members explored the COVID-19-related research activity as well as the impact of the pandemic on preexisting research, related challenges, and coping strategies. The form was distributed via e-mail. Data analyses involved univariate and bivariate methods. Findings. We analyzed 55 responses. A proportion of $58.2 \%$ of respondents reported conducting COVID-19-related research, while 40\% reported that routine research activities were suspended as a result of the pandemic. Major challenges to research in this context were the clinical activity workload, limited access to patients, and research personnel shortage. Coping strategies included adopting remote work and using communication technologies. Conclusion. Despite the many challenges facing the academic researchers to implement COVID-19-related research and to maintain preexisting research activity, there are opportunities to promote academic medical research in the developing world alongside at the global level. Our results should help in documenting and understanding the impact of this pandemic as well as framing appropriate strategies in the future to address similar situations.
\end{abstract}

\section{Introduction}

December 2019 will be marked the beginning of the COVID19 pandemic in China before it spreads to most countries around the world [1]. This crisis has brought up many challenges and caused a profound impact at the global level [2]. The medical and healthcare workforce is at the front line facing the epidemic. They have to provide care to patients and contribute to efforts to contain the pandemic and stop the virus spread.

Research is incremental to tackle the pandemic and its consequences [3-6]. Hence, an exponential increase in the numbers of COVID-19 publications has marked the past few months [7-9]. Different scientific entities have launched initiatives to encourage COVID-19-related research $[10,11]$. Publishers have granted open access to scientific resources related to the pandemic. Many scientific journals have released special issues; some have offered rapid review and publication to encourage manuscript submissions. Funding agencies have also launched calls for research proposals. However, it was challenging for healthcare institutions (including medical schools and teaching hospitals) to implement COVID-19-related research due to the unexpected onset of this crisis and its substantial burden on the healthcare system. Another impact of this pandemic on these institutions could be a disruption of the preexisting research activity.

In Morocco, the first case was notified on March $2^{\text {nd }}$, 2020. A few weeks later, drastic countermeasures were implemented across the country to contain the pandemic [12]. The healthcare system was wholly molded to be prepared for the pandemic. As a consequence, the academic 
medical staff started facing multiple challenges to ensure their threefold mission: healthcare, education, and research. The first mission was to provide care in an unusual, risky, and stressful environment. The second mission consisted of ensuring remote undergraduate, postgraduate, and continuing education. The third mission was to maintain routine research activities as well as to design and conduct new research projects to address COVID-19-related questions. We believe that this pandemic represents an additional challenge to research activity in our context, where it is already difficult to conduct research. Previous literature highlighted the impact of the pandemic on medical teaching institutions and their missions [13-16].

Data on academic research activity by medical faculty staff during this pandemic are scarce. To date, there are no data on this topic from medical teaching institutions in Morocco. Hence, the first aim of the study was to describe the COVID-19 research activity of the medical school faculty members during the pandemic in Marrakesh, Morocco. The second aim was to explore the impact of the pandemic on routine research activity and the coping strategies implemented by researchers.

\section{Methods}

We conducted an online survey in June 2020. The study population consisted of faculty members affiliated to the medical school or the university hospital in Marrakesh, Morocco. A total of 260 individuals were invited to participate. The questionnaire was developed by the authors and then transformed into an online form on Google Forms. The online survey was tested and distributed through a link via e-mails.

The questionnaire consisted of three main sections. The first section gathered sociodemographic and professional data, including sex, age, specialty (medicine/surgery/lab or public health), rank (junior members: assistant/associate professors; senior members: professors), and the number of years as a faculty member. The second section explored the COVID-19-related research activity which included the number of projects, study designs, study populations, data collection methods, production types, funding, and collaboration. The third section explored the impact of the pandemic on research activities and the strategies to cope with it. Respondents were asked whether they could maintain their routine research activity and how significant was the impact of specific aspects linked to the pandemic on this activity. These aspects included clinical activities, limited access to data, limited access to patients, shortage of personnel, lack of material resources, and lack of collaboration. The proposed strategies to maintain research activities included the use of communication technologies, remote work for research personnel, grant writing and submission, and establishing new collaborations. Three open-ended questions aimed to collect additional data on the impact of the pandemic on the research, strategies to maintain research activities, and, more generally, lessons learned from the pandemic context for the research enterprise.
TABle 1: Description of the study participants' characteristics $(n=55)$.

\begin{tabular}{lccc}
\hline Variables & Modalities & $n$ & $\%$ \\
\hline \multirow{2}{*}{ Sex } & Male & 30 & 54.5 \\
& Female & 25 & 45.5 \\
\hline \multirow{2}{*}{ Age groups } & $<40$ years old & 12 & 21.9 \\
& $\geq 40$ years old & 43 & 78.1 \\
\hline \multirow{4}{*}{ Specialty } & Medicine & 25 & 45.5 \\
& Surgery & 18 & 32.7 \\
& Biology/public & 12 & 21.8 \\
Rank & health & & \\
& Assistant professor & 11 & 20.0 \\
& Associate professor & 11 & 20.0 \\
Number of years as a faculty & Professor & 33 & 60.0 \\
member & $<5$ years & 15 & 27.3 \\
\hline
\end{tabular}

TABLE 2: COVID-19-related research activities and production among faculty members.

\begin{tabular}{lcc}
\hline & $N$ & $\%$ \\
\hline Study designs $(n=34)$ & 18 & \\
Case series & 12 & 52.9 \\
Literature reviews & 10 & 35.3 \\
Surveys & 07 & 29.4 \\
Interventional studies & & 20.6 \\
\hline Study populations $(n=34)$ & 24 & \\
COVID-19 patients & 11 & 70.6 \\
Healthcare personnel & 10 & 32.4 \\
General population & 04 & 18.2 \\
COVID-19 contacts & 02 & 11.8 \\
Medical students & 02 & 05.9 \\
Faculty members & & 05.9 \\
\hline Data collection methods $(n=33)$ & 17 & \\
Clinical observation & 16 & 51.5 \\
Medical records review & 08 & 48.5 \\
Online surveys & 04 & 14.5 \\
Face-to-face surveys & 03 & 12.1 \\
Phone surveys & & 05.5 \\
\hline Production (n=31) & 18 & \\
Articles & 09 & 58.1 \\
Research protocols & 06 & 29.0 \\
Oral communications & 04 & 10.9 \\
Posters & & 07.3 \\
\hline
\end{tabular}

This survey was conducted according to the established ethical principles for research. Potential participants were provided with information about the study by e-mail. Then, consenting participants were invited to access the questionnaire via the web-link in the e-mail. Completion of the survey was considered as consent to participate. Data were collected and analyzed anonymously.

Data were extracted as an excel sheet, which was cleaned and then exported to SPSS version 16.0 for Windows. Data were described using frequencies for categorical variables and means and standard deviations for continuous variables. The chi-square test was used to compare percentages. The level of significance was set at $5 \%$. 
TABLE 3: Importance of the impact of COVID-19 pandemic on the preexisting research activities of faculty members.

\begin{tabular}{|c|c|c|c|c|c|}
\hline & \multicolumn{5}{|c|}{ Ratings of the importance of specific aspects related to the pandemic on research activities (\%) } \\
\hline & Not important & $\begin{array}{c}\text { Somehow not } \\
\text { important }\end{array}$ & $\begin{array}{l}\text { Neither important nor not } \\
\text { important }\end{array}$ & Somehow important & Very important \\
\hline Clinical activities workload $(n=50)$ & 10.0 & 10.0 & 16.0 & 24.0 & 40.0 \\
\hline Limited access to data $(n=52)$ & 23.0 & 21.2 & 25.0 & 21.2 & 09.6 \\
\hline Limited access to patients $(n=52)$ & 11.5 & 11.5 & 15.4 & 30.8 & 30.8 \\
\hline Shortage of personnel $(n=51)$ & 15.7 & 11.8 & 13.7 & 29.4 & 29.4 \\
\hline Lack of material resources $(n=51)$ & 15.7 & 11.8 & 33.3 & 23.5 & 15.7 \\
\hline Lack of collaboration $(n=51)$ & 09.8 & 29.4 & 31.4 & 19.6 & 09.8 \\
\hline
\end{tabular}

\section{Results}

We analyzed 55 responses, corresponding to a response rate of $21.2 \%$. The participants' mean age was 44.4 , with values ranging from 33 to 59 years old. Medical specialties were more frequent than surgical and lab or public health ones. Table 1 summarizes the characteristics of the study participants.

Almost two-thirds of respondents $(32 ; 58.2 \%)$ reported conducting COVID-19-related research. The number of projects per individual ranged from 1 to 10 , with a median of 2. Completed projects at the moment of the survey ranged from 0 to 4 . The main study types were case series, surveys, and literature reviews. Diverse populations were targeted by research, including COVID-19 patients, their contacts, and the general population. Other researchers targeted healthcare personnel, medical students, or faculty members. Data were collected mainly through reviewing medical records, followed by clinical observations and face-to-face surveys. Online and phone surveys were used in some cases. Related scientific production consisted mainly of research papers and protocols. Table 2 describes the reported COVID-19-related research activities and output among faculty members.

In most of the cases $(27 ; 79.4 \%)$, researchers did not have or use dedicated funds to conduct their research, whereas very few $(5 ; 14.7 \%)$ used funds from the university or other funding sources. In 21 cases $(65.6 \%)$, respondents reported having had a collaboration with researchers outside of their departments. Of those, 20 (95.2\%) engaged in collaborations with researchers within the same institution, 10 (52.4\%) with researchers from another institution in Morocco, and 9 (42.9\%) with international researchers.

According to $40 \%$ of the responses, routine research activities were suspended as a result of the COVID-19 pandemic. Table 3 describes the importance of the impact of various COVID-19 pandemic aspects on preexisting research activities. The factors that were frequently reported as having a significant effect were the clinical activity workload, limited access to patients, and personnel shortage. Additional comments concerning the impact of the pandemic on research activities by the respondents further emphasized the increased workload resulting in physical and intellectual fatigue and challenges to data collection due to changes imposed on the usual practice.

The strategies used to maintain a certain level of research activity were using communication technologies (36; 75.0\%), remote work for research personnel $(14 ; 29.2 \%)$, grant writing and submission (15; 31.3\%), and finding new collaboration $(14 ; 29.2 \%)$. In response to the open-ended question about the lessons learned from the pandemic concerning the research activities, respondents insisted on the importance of maintaining preexisting research activity and on developing research projects in parallel during the pandemic. Some participants remarked that this period was more convenient to perform research activities. Others pointed to strategies that could promote research such as funding, technical and administrative support, protected research time, collaboration, and use of communication technologies. One participant responded by one term "adaptation."

Overall, there were no statistically significant differences in implementing COVID-19 research or maintaining preexisting research according to specialty, rank, and number of years as a faculty member. One exception was for specialty: medicine, lab, and public health specialists reported more frequent pandemic-related research as compared to their colleagues from surgery specialties $(75.7 \%$ versus $33.3 \%$, respectively; $p=0.02$; Table 4 ).

\section{Discussion}

The COVID-19 pandemic represents an unprecedented health crisis worldwide. This situation had led to a double impact on academic research: it had stimulated research to address the many questions yet it had not answered those concerning the infection. It had also caused significant disruption of the preexisting research activity [17]. Bibliometric analyses have described the available research output [7-9, 18, 19]. The largest numbers of COVID-19-related publications were from the USA and China [19]. Some bibliometric analyses reported that although there are high numbers of COVID-19 publications, they are mainly represented by narrative views, opinions, and case reports [7]. Moreover, Glasziou et al. contended that part of this research is of poor quality because of "time pressure and inadequate research infrastructure" [20]. Odone et al. reported that over $60 \%$ of the publications they examined were opinion papers [21]. The predominance of opinion papers and commentaries was noticed during the early stage of the pandemic, with more original studies published later on [22].

Three out of five respondents to our survey reported having initiated or being involved in COVID-19-related research projects. According to our study, research projects involved diverse study designs and targeted various 
TABLE 4: Factors associated with research activity during the COVID-19 pandemic among faculty members.

\begin{tabular}{|c|c|c|c|c|c|c|c|}
\hline & & \multicolumn{3}{|c|}{$\begin{array}{l}\text { COVID-19-related research } \\
\text { activity }\end{array}$} & \multicolumn{3}{|c|}{$\begin{array}{l}\text { Continuation of routine } \\
\text { research activities }\end{array}$} \\
\hline & & Yes & No & $p$ & Yes & No & $p$ \\
\hline Specialty & $\begin{array}{l}\text { Medicine/Biology/Public health } \\
\text { Surgery }\end{array}$ & $\begin{array}{c}28(75.7) \\
6(33.3)\end{array}$ & $\begin{array}{c}9(24.3) \\
12(66.7)\end{array}$ & 0.020 & $\begin{array}{l}23(62.2) \\
10(55.6)\end{array}$ & $\begin{array}{l}14(37.8) \\
8(44.4)\end{array}$ & 0.639 \\
\hline Rank & $\begin{array}{l}\text { Junior faculty members } \\
\text { Senior faculty members }\end{array}$ & $\begin{array}{l}12(54.5) \\
22(66.7)\end{array}$ & $\begin{array}{l}10(45.5) \\
11(33.3)\end{array}$ & 0.365 & $\begin{array}{l}14(63.6) \\
19(57.6)\end{array}$ & $\begin{array}{c}8(36.4) \\
14(42.4)\end{array}$ & 0.653 \\
\hline Number of years as a faculty member & $\begin{array}{l}<5 \text { years } \\
\geq 5 \text { years }\end{array}$ & $\begin{array}{c}8(53.3) \\
26(65.0)\end{array}$ & $\begin{array}{c}7(46.7) \\
14(35.0)\end{array}$ & 0.428 & $\begin{array}{l}10(66.7) \\
23(57.5)\end{array}$ & $\begin{array}{c}5(33.3) \\
17(42.5)\end{array}$ & 0.537 \\
\hline
\end{tabular}

populations. Descriptive studies and reviews represented the most frequent research designs. The main research outputs were articles and research protocols. In PubMed, on September $1^{\text {st }}, 98$ citations were found for COVID-19 or SARSCoV-2 in the title or abstract and Morocco in affiliation. Of those, there were sixteen letters, fifteen reviews, and four case reports. It is possible that other studies are still ongoing or are not indexed in Medline. This quick search was meant to provide some insight into the production from Morocco in the form of publications considering the example of a major database for health and biomedical sciences. As many research projects were still ongoing, higher evidence research output should be expected in the future in our context as well.

Target populations for these research projects were mainly COVID-19 patients and their contacts. Other populations were targeted due to the broad impact of the pandemic on various groups of society. Healthcare professionals represented another interesting group, with the aim of capturing their experience and perspectives of the pandemic and its management. Due to drastic distancing measures, all in-campus educational activities were suspended, and important efforts were invested in ensuring distance teaching activities. In this context, the students and faculty members themselves became a target population for the study of the impact and strategies during the pandemic.

During the pandemic, there was a shift in the communication channels as distance and online communication took over in-person communication in most domains and aspects [23]. As part of the restrictive public health countermeasures, it has been recommended to use new technologies and to privilege remote work whenever it was possible. In our survey, medical records review and clinical observations were still the main methods of data collection even though other distance and online tools were used. In our context, these tools could be more useful for teamwork and mentoring purposes than for data collection. The use of these tools for data collection will depend on the target population as it may be more relevant and useful among students or pairs than among patients or the general population.

Noteworthy, very few respondents used dedicated funds for their research. This is not surprising due to the scarcity of funding sources for research in developing countries and the many challenges to raise funds. In a recent review, Ibrahim et al. outline the situation and challenges to the COVID-19 research in the Middle East and North Africa region [24].

On the other hand, a majority of researchers who conducted a COVID-19-related project had a collaboration with other researchers outside their departments, including international collaborations. This is a positive finding as previous literature has emphasized that insufficient research collaboration between countries results in limited research efficiency [8]. Moreover, the lack of collaboration and communication was reported as a cause of useless duplication and "waste" in COVID-19 research [20]. Nonetheless, it is not possible to assess the impact and efficiency of this collaboration based on this survey.

Forty percent of the respondents had to suspend their preexisting research activity due to the pandemic. The COVID-19 pandemic had a profound impact on healthcare systems in general, with drastic changes in healthcare provision and education. Indeed, teaching hospitals in Morocco were in the front line of the response strategy and were reoriented to manage COVID-19 patients. This has disrupted routine activities, including the provision of care, teaching, and research. Most of these activities were suspended for two main reasons: to ensure proper preparedness to manage an unexpected flow of patients and to protect personnel, students, patients, and research participants against contamination. The authors have described the impact of the pandemic on research activities which were suspended as a result of containment measures [25]. Wigginton et al. estimated that the pandemic has halted more than $80 \%$ of on-site research in 6 US universities [26].

In our study, those who could maintain their research activity reported challenges due to this health crisis. More specifically, research activities were suspended for practical reasons such as clinical workload, limited access to patients due to containment measures, and personnel shortage. It is also possible that research interests and capabilities in this particular period are different and dependent on various factors. One factor (specialty) was significantly associated with the conduct of COVID-related research. Other factors including the setting and the stage of the pandemic could determine the impact and guide coping strategies [27].

Strategies to promote or maintain research activity in the context of the current pandemic included the use of communication technologies and remote work. These strategies have been proposed or implemented in various 
settings to allow for continuing teaching and research activities [16, 23, 28]. Moreover, these tools were found useful for healthcare activities and teaching [14]. Indeed, academic medical institutions need to deploy every strategy with a potentially positive impact on their "tripartite mission" [15]. Recent literature described several strategies that were implemented to select "critical" research projects to be continued and possible changes to their conduct [29-32]. The adopted strategies differed considering the research area and setting. Yet, some shared perspectives included preserving the productivity and protecting the personnel primarily by promoting remote work [33]. In this context, web-based surveys have become popular [34] and were used whenever relevant.

This study sheds light on a topic of growing interest in the context of this pandemic. Research is a key activity to understand and tackle this situation. We involved a key population for health research, which is the academic medical staff whose mission includes research alongside healthcare and education. The response rate in this study was low. This could be linked to the stressful work environment and less interest in research during this period. It could also be explained by the data collection method using an online questionnaire. We recruited a volunteer sample, which can lead to a selection bias. It is possible that people who were more interested in the research were keener to participate in this survey, which may imply that proportions of respondents reporting conducting COVID-related research $(58.2 \%)$ or maintaining their preexisting research activity (60\%) could be overestimated.

\section{Conclusion}

To sum up, we explored two effects of the COVID-19 pandemic on research activity among medical faculty. On the one hand, this crisis stimulated research to address related questions and was implemented in a challenging context. On the other hand, it disrupted the prexisting research activity due to significant changes in institutions and professionals' routines. It is not possible to continue with all research projects during this health crisis. Still, it is quite possible to adapt our strategies to make the best out of available resources and opportunities. While our results may not be readily generalizable to every context, they contribute substantially to documenting the impact of this crisis in our context. It is of utmost importance to strengthen the academic research environment to face similar situations in the future, especially in developing countries.

\section{Data Availability}

The data used in the study are available from the corresponding author upon reasonable request.

\section{Conflicts of Interest}

The authors declare that they have no conflicts of interest.

\section{References}

[1] T. Acter, N. Uddin, J. Das, A. Akhter, T. R. Choudhury, and S. Kim, "Evolution of severe acute respiratory syndrome coronavirus 2 (SARS-CoV-2) as coronavirus disease 2019 (COVID-19) pandemic: a global health emergency," Science of the Total Environment, vol. 730, Article ID 138996, 2020.

[2] J. Whitworth, "COVID-19: a fast evolving pandemic," Transactions of The Royal Society of Tropical Medicine and Hygiene, vol. 114, no. 4, pp. 241-248, 2020.

[3] A. Haleem, M. Javaid, R. Vaishya, and S. G. Deshmukh, "Areas of academic research with the impact of COVID-19," The American Journal of Emergency Medicine, vol. 38, no. 7, pp. 1524-1526, 2020.

[4] E. A. Holmes, R. C. O'Connor, V. H. Perry et al., "Multidisciplinary research priorities for the COVID-19 pandemic: a call for action for mental health science," The Lancet Psychiatry, vol. 7, no. 6, pp. 547-560, 2020.

[5] M. Ballard, D. L. Swerdlow, and L. Finelli, "Defining the epidemiology of Covid-19-studies needed," New England Journal of Medicine, vol. 382, no. 13, pp. 1194-1196, 2020.

[6] K.-S. Yuen, Z.-W. Ye, S.-Y. Fung, C.-P. Chan, and D.-Y. Jin, "SARS-CoV-2 and COVID-19: the most important research questions," Cell and Bioscience, vol. 10, p. 40, 2020.

[7] S. B. S. Kambhampati, R. Vaishya, and A. Vaish, "Unprecedented surge in publications related to COVID-19 in the first three months of pandemic: a bibliometric analytic report," Journal of Clinical Orthopaedics and Trauma, vol. 11, pp. S304-S306, 2020.

[8] Z. Tao, S. Zhou, R. Yao et al., "COVID-19 will stimulate a new coronavirus research breakthrough: a 20-year bibliometric analysis," Annals of Translational Medicine, vol. 8, no. 8, p. $528,2020$.

[9] B. X. Yang, G. H. Ha, L. H. Nguyen et al., "Studies of novel coronavirus disease 19 (COVID-19) pandemic: a global analysis of literature," International Journal of Environmental Research and Public Health, vol. 17, no. 11, 2020.

[10] J. A. Helliwell, W. S. Bolton, J. R. Burke, J. P. Tiernan, D. G. Jayne, and S. J. Chapman, "Global academic response to COVID-19: cross-sectional study," Learned Publishing, vol. 33, no. 4, pp. 385-393, 2020.

[11] J. J. Lee and J. P. Haupt, "Scientific globalism during a global crisis: research collaboration and open access publications on COVID-19," Higher Education, pp. 1-18, 2020.

[12] M. Sebbani, L. Adarmouch, A. Mansouri, and M. Amine, "Evolution of COVID-19 in relation to public health countermeasures in Morocco," Open Journal of Epidemiology, vol. 10, no. 2, pp. 187-194, 2020.

[13] D. Almaghaslah and A. Alsayari, "The effects of the 2019 novel coronavirus disease (COVID-19) outbreak on academic staff members: a case study of a pharmacy school in Saudi Arabia," Risk Management and Healthcare Policy, vol. 13, pp. 795-802, 2020.

[14] B. Ashokka, S. Y. Ong, K. H. Tay, N. H. W. Loh, C. F. Gee, and D. D. Samarasekera, "Coordinated responses of academic medical centres to pandemics: sustaining medical education during COVID-19," Medical Teacher, vol. 42, no. 7, pp. 762-771, 2020.

[15] T. Standiford, A. G. Shuman, D. Fessell, M. J. Brenner, and C. R. Bradford, "Upholding the tripartite mission in times of crisis: purpose and perseverance in the COVID-19 pandemic," Otolaryngology-Head and Neck Surgery, vol. 163, no. 1, pp. 54-59, 2020. 
[16] M. B. Omary, J. Eswaraka, S. D. Kimball, P. V. Moghe, R. A. Panettieri, and K. W. Scotto, "The COVID-19 pandemic and research shutdown: staying safe and productive," Journal of Clinical Investigation, vol. 130, no. 6, pp. 2745-2748, 2020.

[17] L. Zhang, W. Zhao, B. Sun, Y. Huang, and W. Glänzel, "How scientific research reacts to international public health emergencies: a global analysis of response patterns," Scientometrics, pp. 1-27, 2020.

[18] M. Haghani, M. C. J. Bliemer, F. Goerlandt, and J. Li, "The scientific literature on Coronaviruses, COVID-19 and its associated safety-related research dimensions: a scientometric analysis and scoping review," Safety Science, vol. 129, Article ID 104806, 2020.

[19] S. H. Zyoud and S. W. Al-Jabi, "Mapping the situation of research on coronavirus disease-19 (COVID-19): a preliminary bibliometric analysis during the early stage of the outbreak," BMC Infectious Diseases, vol. 20, no. 1, p. 561, 2020.

[20] P. Glasziou, D. G. Altman, P. Bossuyt et al., "Reducing waste from incomplete or unusable reports of biomedical research," The Lancet, vol. 383, no. 9913, pp. 267-276, 2014.

[21] A. Michie, S. Salvati, L. Bellini et al., "The runaway science: a bibliometric analysis of the COVID-19 scientific literature," Acta Bio Medica Atenei Parmensis, vol. 91, no. 9-S, pp. 34-39, 2020.

[22] J. Nowakowska, J. Sobocińska, M. Lewicki, Ż. Lemańska, and P. Rzymski, "When science goes viral: the research response during three months of the COVID-19 outbreak," Biomedicine \& Pharmacotherapy, vol. 129, Article ID 110451, 2020.

[23] A. M. Schwartz, J. M. Wilson, S. D. Boden, T. J. J. Moore, T. L. J. Bradbury, and N. D. Fletcher, "Managing resident workforce and education during the COVID-19 pandemic: evolving strategies and lessons learned," JBJS Open Access, vol. 5, no. 2, p. e0045, 2020.

[24] H. Ibrahim, A. M. Kamour, T. Harhara, W. H. Gaba, and S. C. Nair, "Covid-19 pandemic research opportunity: is the Middle East \& North Africa (MENA) missing out?," Contemporary Clinical Trials, vol. 96, Article ID 106106, 2020.

[25] F. Klont and G. Hopfgartner, "Bioanalytical research and training in academia during the COVID-19 pandemic," Bioanalysis, vol. 12, no. 17, 2020.

[26] N. S. Wigginton, R. M. Cunningham, R. H. Katz et al., "Moving academic research forward during COVID-19," Science, vol. 368, no. 6496, pp. 1190-1192, 202012.

[27] A. Zuber, M. Katsoulis, A. G. Lai et al., "Clinical academic research in the time of Corona: a simulation study in England and a call for action," PLoS One, vol. 15, no. 8, Article ID e0237298, 2020.

[28] A. Chowdhry, P. Kapoor, and D. B. Popli, "Strengthening health care research and academics during and after COVID19 pandemic- an Indian perspective," Journal of Oral Biology and Craniofacial Research, vol. 10, no. 4, pp. 343-346, 2020.

[29] M. Marcum, N. Kurtzweil, C. Vollmer et al., "COVID-19 pandemic and impact on cancer clinical trials: an academic medical center perspective," Cancer Medicine, vol. 9, no. 17, pp. 6141-6146, 2020.

[30] N. M. Noor, A. L. Hart, P. M. Irving, S. Ghosh, M. Parkes, and T. Raine, "Clinical trials (and tribulations): the immediate effects of COVID-19 on IBD clinical research activity in the United Kingdom," Journal of Crohn's and Colitis, 2020.

[31] A. Vagal, S. B. Reeder, D. K. Sodickson, V. Goh, Z. M. Bhujwalla, and E. A. Krupinski, "The impact of the COVID-19 pandemic on the radiology research enterprise: radiology scientific expert panel," Radiology, vol. 296, no. 3, pp. E134-E140, 2020.

[32] E. C. Verna, M. Serper, J. Chu et al., "Clinical research in hepatology in the COVID-19 pandemic and post-pandemic era: challenges and the need for innovation," Hepatology (Baltimore, Md.), vol. 72, no. 5, pp. 1819-1837, 2020.

[33] A. Goyal, P. Kerezoudis, Y. U. Yolcu et al., "Letter to the editor: survey of academic U.S. Programs regarding the impact of the COVID-19 pandemic on clinical practice, education, and research in neurosurgery," World Neurosurgery, vol. 140, pp. 476-478, 2020.

[34] R. B. D. Bendok and R. B. D. Boni, "Web surveys in the time of COVID-19," Cadernos de Saúde Pública, vol. 36, no. 7, 2020. 